\title{
La littérature inférieure : trois présupposés interprétatifs
}

Lower Literature: Three Interpretative Presuppositions

\section{Urszula Glensk}

Traducteur : Anna Saignes

\section{(2) OpenEdition \\ Journals}

Édition électronique

URL : https://journals.openedition.org/recherchestravaux/3564

DOI : 10.4000/recherchestravaux.3564

ISSN : 1969-6434

Éditeur

UGA Éditions/Université Grenoble Alpes

Édition imprimée

ISBN : 978-2-37747-301-4

ISSN : 0151-1874

Référence électronique

Urszula Glensk, «La littérature inférieure : trois présupposés interprétatifs », Recherches \& Travaux [En ligne], 98 | 2021, mis en ligne le 28 juin 2021, consulté le 09 août 2021. URL : http://

journals.openedition.org/recherchestravaux/3564; DOI : https://doi.org/10.4000/recherchestravaux 3564

Ce document a été généré automatiquement le 9 août 2021.

(c) Recherches \& Travaux 


\title{
La littérature inférieure : trois présupposés interprétatifs
}

\author{
Lower Literature: Three Interpretative Presuppositions
}

\author{
Urszula Glensk
}

Traduction : Anna Saignes

1 Dans les études littéraires polonaises, le reportage a été l'objet de très nombreuses études théoriques. Pourtant, toutes ces études ont un air de famille : elles débutent généralement par une explication du sens du verbe latin reportare, procèdent ensuite à des classifications et énumèrent des définitions du genre. L'abondance de formules définitionnelles aboutit à un phénomène que Franklin Ankresmit appelle " polychromie interprétative ${ }^{1}$ » et qui finit par inhiber l'autonomie herméneutique du lecteur. D'autre part, lorsqu'on confronte la théorie avec la vitalité narrative des textes, on s'aperçoit que la théorie ne met pas à la disposition des critiques d'outils efficaces. Ainsi l'ouvrage qui a obtenu en 2019 le prix Kapuściński², réservé au reportage, Dom z dwoma wieżami ${ }^{3}$ [La maison aux deux tours] de Maciej Zaremba Bieławski, ne correspond pas vraiment aux définitions habituelles du genre. Il s'agit plutôt d'une autobiographie où le récit de soi constitue un prétexte pour raconter les expériences de trois générations : le grand-père juif, les parents et l'auteur. Cependant l'abondance des détails et la puissance du récit à ressusciter le passé pour le lecteur transforment l'autobiographie en une ample fresque sociale, ce qui le rapproche du reportage. Le reportage - et cela ne fait que lui ajouter de la valeur - échappe aux règles simples, déplace les frontières entre analyse impartiale et engagement personnel, entre compterendu journalistique et création littéraire.

2 On s'accorde pour dire que dans le reportage, le monde représenté reste en relation étroite avec une expérience réelle. Le reportage raconte des événements et des situations que l'auteur a expérimentés, auxquels il a participé ou sur lesquels il s'est documenté en recueillant le récit d'un acteur ou d'un témoin. Dans le reportage, l'auteur réel est identique au narrateur. En d'autres mots, la personne dont le nom figure sur la couverture du livre est aussi celle qui prend en charge le récit, de manière discrète ou exhibée. La personne qui signe l'œuvre de son nom est en même temps celle 
qui s'exprime dans le texte. L'identité de l'auteur et du narrateur constitue ainsi une partie du "pacte du reportage", qui postule le caractère authentique du récit et l'intention, qui le sous-tend, de raconter le réel. Mais nous nous heurtons alors à une autre difficulté interprétative : qu'est-ce qui fait la différence entre le reportage ainsi défini d'une part et de l'autre, les écrits à caractère scientifique, les informations journalistiques ou encore les souvenirs, autant de formes qui sont aussi animées par le souci de référentialité et d'authenticité ? La différence reposerait sur le fait que les auteurs de reportages utilisent un ensemble de procédés littéraires, notamment stylistiques, élaborés par la littérature de fiction. Cette vision a d'ailleurs donné lieu à la formule dépréciative selon laquelle le reportage serait « le bâtard de la littérature ${ }^{4}$ ».

Il est donc difficile d'enfermer le reportage dans une définition qui rendrait compte de tous ses aspects. On s'efforce de masquer l'impuissance théorique au moyen de la notion de "genre limite ${ }^{5}$ ", en posant que le reportage est une forme mixte aux contours imprécis. L'auteur bulgare Guéorgui Gospodinov, également théoricien, pour mettre fin aux débats sur les genres, a affirmé : «Les genres purs ne m'intéressent guère. Le roman n'est pas un Aryen ${ }^{6}$.» On peut en dire autant du reportage.

Je ne vais donc pas m'efforcer de tracer des lignes de partage entre la fiction et l'écriture documentaire. Je voudrais en revanche pointer quelques dénominateurs communs aux réflexions sur le reportage. Mon expérience de lectrice me suggère en effet que la littérature du réel est invérifiable, qu'elle suppose déterritorialisation et semi-perspectivisme.

\section{L'invérifiabilité}

5 L'idée est répandue parmi les critiques que les faits racontés dans les reportages peuvent être confirmés ou réfutés, à l'aide d'outils analytiques adéquats. Les outils de la critique littéraire ou de la critique historique appliquée à la littérature peuvent certes permettre de mettre en question certains éléments du récit, montrer certaines inexactitudes et déformations, résultant d'une perception erronée, d'une mauvaise analyse, d'un souvenir brouillé.

6 Il est assez facile de repérer des erreurs dans les informations ou les chiffres. Ainsi dans son reportage sur Irena Sendler ${ }^{7}$, qui a sauvé des enfants juifs du ghetto de Varsovie, Anna Bikont avance que la somme consacrée par le gouvernement polonais en exil à Londres en mars 1943 aux opérations organisées par Żegota (commission d'aide aux juifs qui opérait en Pologne pendant l'occupation) s'élève à 270 millions de zlotys. En réalité cette somme était de 270000 zlotys. Un lecteur attentif peut cependant déceler cette erreur s'il lit la note de bas de page correspondante qui donne le bon chiffre. Dans un cas comme celui-ci, l'erreur ne disqualifie pas la valeur du livre, un livre scrupuleusement documenté. Si certaines déformations, intentionnelles ou non, peuvent être repérées lors d'une lecture critique, la vérification exhaustive est impossible. Beaucoup d'informations, dans le reportage, proviennent d'entretiens avec des témoins, du savoir d'un individu, d'un récit éphémère. Le lecteur peut miser sur la fiabilité du reporter, ou alors procéder par recoupements avec d'autres reportages ou documents.

7 Plusieurs procédés renforcent la crédibilité du récit. Les histoires racontées au reporter par des individus peuvent donner une impression d'authenticité grâce au langage varié (sociolectes, idiolectes, régionalismes, jargons professionnels, etc.) des locuteurs, 
lorsque les particularités de celui-ci sont conservées dans le texte. On peut ici prendre comme exemple le recueil de témoignages d'enfants sur la Shoah dans Je reviendrai quand tu dormiras. Conversations avec les enfants de l'Holocauste de Patrycja Dołowy ${ }^{8}$. Ce livre de reportage contient plusieurs dizaines de témoignages qui possèdent chacun des caractéristiques langagières propres. La crédibilité du récit est également bien servie par la multiplication des faits et par l'organisation de ceux-ci en enchaînement logique. C'est là ce qui caractérise les reportages de Cezary Łazarewicz centrés sur des affaires criminelles. C'est aussi le cas du livre consacré par Åsne Seierstad à Anders Breivik, le responsable des attentats du 22 juillet 2011 en Norvège ${ }^{9}$. Le même effet de véracité peut être obtenu par une accumulation de détails lors de descriptions de lieux situés très loin du monde du lecteur. C'est ainsi qu'est construit le récit de Ben Rawlence, City of Thorns $^{10}$ [ La cité des épines], consacré au camp de réfugiés de Dadaab au Kenya. Dans l'épilogue l'auteur commente son propre travail ainsi : «[...] dans certains cas, j'ai été le témoin oculaire des événements. Dans d'autres je les ai reconstruits grâce aux entretiens avec des personnes qui y avaient participé, en confrontant leurs récits avec des témoignages et des rapports officiels auxquels j'ai pu avoir accès ${ }^{11}$. Il affirme qu'il a mené ces entretiens en swahili ou en anglais ou encore avec l'aide de traducteurs. Il a dévoilé l'identité de certains, il l'a changée pour d'autres, afin de ne pas les mettre en danger. Ce dernier procédé, consistant à camoufler les véritables identités des personnes impliquées, est typique des littératures du réel contemporaines. De nombreux reporters y ont recouru pour rendre plus crédibles des récits contenant une dose importante de fiction. Nous, lecteurs, ne pourrons jamais le vérifier.

8 Il arrive que les reporters effacent scrupuleusement l'identité de leurs personnages. Après la publication, qui a fait grand bruit, du reportage de Mariusz Szczygieł sur une femme qui a tué son enfant et qui, après avoir purgé sa peine, est devenue enseignante et experte des questions de l'éducation, l'auteur a expliqué :

En plus du changement de l'identité du personnage, et de celle des personnes qui étaient en relation avec elle, j'ai décidé de transformer chaque citation qu'il était possible de retrouver sur internet ou à la Bibliothèque Nationale, afin que personne ne puisse identifier les auteurs de ces citations, c'est-à-dire les proches d'Ewa T. C'est pourquoi mon texte n'est pas accompagné d'une liste de sources. Ce qui y est dit est vrai, mais seulement du point de vue du fond. Les mots sont différents. Pour les mêmes raisons, je n'ai pas précisé que le reportage que je cite a été écrit par Barbara Seidler parce qu'il mentionne la véritable identité d'Ewa T. [...]. Toutes ces opérations, grâce auxquelles j'ai voulu protéger la personne, étaient très naïves. En réalité, quelques minutes après la publication du texte sur internet, dans la version numérique de Duży Format (Grand format ${ }^{12}$ ), le nom de la femme en question a été retrouvé par les internautes ${ }^{13}$.

9 Les doutes quant à la véracité du récit sont souvent impossibles à lever. Il y a là un paradoxe : plus les événements racontés sont étonnants et atypiques plus le texte est attirant mais en même temps plus il est difficile d'y croire. Parfois l'hétérogénéité stylistique permet d'apercevoir des failles dans les paroles rapportées et donne l'impression qu'elles ont été transformées afin de mieux s'adapter au discours du livre. Dans le reportage Płuczki ${ }^{14}$ [Les épurateurs] de Paweł Piotr Reszka, des gens racontent comment ils ont fouillé la terre autour des camps de la mort pour y chercher de l'or. On y entend cependant parfois des phrases qui semblent tirées de l'ouvrage d'un moraliste. Il est difficile de croire à leur authenticité.

10 Ainsi, le pacte du reportage suppose que l'auteur est fiable mais n'exclut pas le scepticisme interprétatif. Nos efforts pour décider si quelque chose est vrai ou inventé 
ne conduisent, la plupart du temps, nulle part. L'impossibilité de la vérification découle également des limites de la perception et de la connaissance, de l'impossibilité de reconstituer les faits en eux-mêmes, de la remise en cause de l'esthétique de la représentation par Roland Barthes dans "L'effet de réel ${ }^{15}$ ». Elle est aussi liée à l'inexistence de "faits de mémoire purs", admise par tous les spécialistes des neurosciences. Le savoir sur les événements demeure dans les consciences des acteurs et des témoins mais il n'est qu'une représentation de ces événements dans les mémoires fragiles d'individus, et non la réalité. Enfin, cette impossibilité est également liée au fait que dans les esprits des acteurs et des témoins, les souvenirs ne rendent pas nécessairement compte de la réalité exacte mais souvent de la manière dont celle-ci est représentée dans la mémoire individuelle, incertaine, changeante, fugace, chargée d'affectivité et d'écrans idéologiques.

\section{La déterritorialisation}

11 Le deuxième dénominateur commun aux reportages est la "déterritorialisation ${ }^{16}$ ", c'est-à-dire l'éloignement des reporters par rapport à leur propre territoire. En effet, les origines du reportage sont à chercher dans le récit de voyage et la périégèse. La découverte et la description de lieux lointains constituent sans aucun doute le geste fondateur du reportage et en sont toujours une variante répandue. Antoni Słonimski dans ses Lettres d'Amérique, publiées dans les années 1930 dans l'hebdomadaire Wiadomości Literackie, affirme que lorsqu'il écrit des reportages, il se fait " périscope » :

J'essaye de décrire ce que je fais [...] je suis un outil qui sert à quelque chose de bien précis. Je suis une sorte de périscope qui permet à une petite poignée d'êtres humains habitant l'autre côté du globe de voir les gens qui se trouvent ici. J'ajoute que je suis un périscope un peu rebelle ${ }^{17}$.

Il observe et note, grâce à quoi les lecteurs peuvent observer à leur tour.

La position de l'observateur situé à la périphérie donne au reporter la possibilité de ne pas être empêtré dans le local, de demeurer «au-dessus ", " entre » la possibilité de collectionner librement des impressions, sans prendre parti. Les voyageurs ne sont pas les seuls observateurs extérieurs. Ce rôle peut aussi être joué par les correspondants de guerre, qui se rendent là où sévit la violence et où il faut témoigner de ses conséquences. Ils sont prêts à se déplacer, à vivre des expériences éprouvantes et leurs conséquences psychologiques. La déterritorialisation est un outil permettant de s'approcher d'une impossible perspective neutre. Elle augmente la fiabilité du texte. Voici quelques exemples de reportages de ce type : la correspondance de guerre d'Åsne Seierstad, L'Ange de Grozny ${ }^{18}$, les livres de Martín Caparrós, comme La Faim ${ }^{19}$ ou le récit de Maciej Wasielewski sur un sinistre épisode de l'histoire de l'̂lle Pitcairn ${ }^{20}$. Le privilège de la déterritorialisation est également celui des reporters qui ont relevé le défi de décrire l'altérité lorsqu'ils se rendent dans les refuges pour sans-abris, les institutions fermées, les abattoirs. On peut donner comme exemple le livre de Drauzio Varella, Le dernier cercle $e^{21}$, sur la plus dangereuse prison du Brésil, où il a soigné des malades. La posture déterritorialisée a été également adoptée par Arlie Russell Hochschild, une sociologue de Berkley, auteure de Strangers in their own land: Anger and Mourning on the American Right ${ }^{22}$. L'auteure a interrogé les habitants de la Louisiane sur les motivations qui les ont amenés à voter pour Donald Trump. Elle met en évidence les points de divergence, les différences culturelles, tout en restant la porte-parole de ses propres 
valeurs. Dans un monde qui lui est étranger, elle cherche une réponse à des conflits dans lesquels elle-même est enlisée.

Le processus de la déterritorialisation nécessite qu'on s'écarte de son propre milieu, au sens géographique comme identitaire du terme. De cette façon la littérature documentaire est capable de raconter l'hétérogénéité du monde réel.

\section{Le semi-perspectivisme}

L'éloignement du reporter par rapport à son territoire permet à celui-ci de cheminer vers une neutralité qu'il souhaite atteindre, sans jamais y parvenir vraiment. Cependant dans la réflexion sur l'écriture du reportage apparaissent également des catégories comme l'empathie, l'engagement, l'expérience, la compréhension, la sensibilité. Une partie du travail du reporter consiste à écouter le récit des autres. Cela demande une façon spécifique d'aborder le monde : le semi-perspectivisme. Le regard du reporter rencontre celui de son personnage à mi-chemin.

Dans l'art du reportage la capacité à écouter ce que les autres ont à dire est primordiale, même si ceux qui racontent sont connus pour avoir dépassé les limites de ce qui est permis par les normes éthiques et sociales. Il suffit de mentionner le reportage de l'auteure croate, Slavenka Drakulić, qui a raconté les vies de Ratko Mladić, Slobodan Miloševic et d'autres criminels jugés à La Haye ${ }^{23}$. Le titre du reportage, Ils ne feraient pas de mal à une mouche, renvoie à la pensée d'Hannah Arendt ${ }^{24}$. Le récit de Drakulić, composé de plusieurs strates, montre que le reportage, grâce à son pouvoir de démasquer les processus politiques, sociaux ou historiques, peut avoir une signification sociale et une efficacité pour changer les esprits et les comportements. Il en est ainsi dans King Leopold's Rule in Africa, le reportage d'Edmund Dene Morel ${ }^{25}$, qui a attiré l'attention de l'opinion sur la brutalité de la colonisation du Congo par les Belges. En 1933, le reportage d'Ewa Szelburg-Zarembina intitulé Lavez vos fruits ${ }^{26}$, a sauvé de la peine de mort plusieurs paysans accusés d'avoir cherché à déclencher une révolution communiste en Pologne. Le reportage a remué l'opinion et amené plusieurs intellectuels à exprimer leur indignation face à la procédure d'exception mise en œuvre. C'est grâce au reportage que la peine de mort requise n'a pas été prononcée. L'ouvrage d'Hannah Arendt, Eichman à Jérusalem, est devenu un classique de la réflexion sur la Shoah. Jan Tomasz Gross, dans Les Voisins ${ }^{27}$, grâce à des témoignages de survivants, a mis en évidence la participation des Polonais au pogrom de Jedwabne (le 10 juin 1941) et a ainsi ouvert la voie aux recherches sur le rôle des Polonais dans l'extermination des juifs en Pologne durant la Seconde Guerre mondiale.

La pratique du reportage nécessite, de la part des auteurs, une aptitude au décentrement, une capacité à écouter les autres, même lorsque leur récit est affligeant ou traumatisant pour celui qui l'écoute. Avant que le reporter n'écrive son premier paragraphe, il doit écouter, observer, comprendre, entrer en «synchronie émotionnelle ${ }^{28}$ » avec le protagoniste de son récit, accepter de regarder la souffrance de l'autre, comme l'a si bien décrit $\mathrm{S}$. Sontag ${ }^{29}$. La réflexion empathique prenant pour objet la souffrance des autres et de sociétés entières semble constituer une des principales valeurs de cette pratique d'écriture.

L'entreprise du reporter s'expose à deux risques: un risque émotionnel et un risque physique. Le correspondant de guerre Ed Vuilliamy reconnaît que parfois « son regard se brouille de terreur » et que son " corps désobéit ${ }^{30}$ ». Ces risques concernent non 
seulement les reporters de guerre mais aussi ceux qui utilisent la méthode du reportage provoqué. Parmi ces derniers, le reporter allemand Günter Wallraff est sans doute le plus connu grâce à ses entreprises audacieuses. En 1985-1986, il s'est fait passer pour un immigré turc prêt à faire tout travail afin de dénoncer les conditions de travail inhumaines que subissent les travailleurs immigrés en Allemagne ${ }^{31}$. Il a aussi endossé le rôle du prisonnier politique en Grèce dans les années 1970 et s'est fait passer pour un sans-abri endormi sur un banc par une nuit glaciale ${ }^{32}$. Il admet qu'il a souvent eu peur et ajoute que « dans ces situations il entend une voix intérieure [qui lui dit] : même si je meurs, cela aura un sens, parce que d'autres vont en tirer profit ${ }^{33}$.»

La tâche du reporter est de collectionner des "événements représentatifs ", selon la formule de Leo Marx, c'est-à-dire des épisodes caractéristiques d'une époque, reflétant une façon de penser et "légitimant des façons de voir les choses ordinaires ${ }^{34}$ ». La description des "événements représentatifs" est une mise à nu. Cela implique une relation complexe avec ceux qui racontent leurs histoires au reporter. Le semiperspectivisme facilite le contact, rend possible le passage du jugement de valeurs vers la compréhension. C'est un outil indispensable au reporter.

Quant à ce qui se passe entre protagonistes et reporters une fois le livre publié, ces derniers rechignent à en parler. Dans son reportage sur les enfants enlevés par Joseph Kony et incorporés de force dans l'Armée de résistance du seigneur, le reporter polonais Wojciech Jagielski transcrit ainsi sa conversation avec l'éducatrice d'un centre de réinsertion :

«D'autres que toi sont venus ici. Ils posaient des questions aux enfants et puis ils partaient et au moins c'était clair. Et toi, tu es revenu. Je pensais que ça allait se passer autrement. Pourquoi tu es revenu? Pour le travail! Pour le travail! C'est ce que je dois dire à Samuel s'il me pose la question? Tu es entré dans notre vie et maintenant tu as peur. Tu as peur de quoi ? De t'être trop rapproché de nous?» J'ai rassemblé mes affaires pour partir ${ }^{35}$.

La nécessité de s'immiscer constamment dans la vie des autres n'est pas sans conséquences pour les reporters. Ils sont nombreux à parler d'empathie émotionnelle ou de résonance affective et à évoquer les difficultés psychologiques qu'entraine la contagion par la souffrance des victimes.

Les catégories que j'ai proposées - l'invérifiabilité, la déterritorialisation, le semiperspectivisme - tracent un triangle interprétatif qui permet de saisir la spécificité des textes où les auteurs ont l'ambition de transformer la réalité en récit. Les périphéries des genres, les expériences formelles, les frontières floues : ces notions dessinent un espace où viennent se loger les reportages. 


\section{NOTES}

1. F.R. Ankersmit, «Historiografia i postmodernism » [« Historiography and postmodernism », History and Theory, vol. 28, $\mathrm{n}^{\circ} 2,1989$ ], dans Ryszard Nycz (éd.), Postmodernizm. Antologia przekładów [Le postmodernisme. Anthologie des traductions], Cracovie, 1998, p. 147.

2. Prix récompensant chaque année le meilleur reportage, polonais ou étranger.

3. M. Zaremba Bielawski, Dom z dwoma wieżami, Varsovie, Karakter, 2018.

4. W. Kubacki, «Zmierzch reportażu» [«Le crépuscule du reportage»], Tygodnik Ilustrowany [L’hebdomadaire illustré], 1937, $\mathrm{n}^{\circ} 37$.

5. H. M. Małgowska, «Gatunki reportażowo-dziennikarskie okresu dwudziestolecia (próba typologii)» [«Les genres du reportage et du journalisme dans l'entre-deux-guerres (essai de typologie) »], Z dziejów form artystycznych $w$ literaturze polskiej. $Z$ teorii i historii literatury [Histoire des formes dans la littérature polonaise], t. 1, IBL PAN, 1963, p. 193-200.

6. G. Gospodinov, Physique de la mélancolie, traduit du bulgare par Marie Vrinat-Nikolov, Paris, Intervalles, 2015, p. 196 [ФИЗИКА НА ТЪГАТА, 2011].

7. A. Bikont, Sendlerowa. W ukryciu [Irena Sendler. Dans la clandestinité], Czarne, Wołowiec, 2017 (non traduit en français).

8. P. Dołowy, WrocĘ, gdy będziesz spała. Rozmowy z dziećmi Holokaustu [Je reviendrai quand tu dormiras. Conversation avec les enfants de l'Holocauste], Czarne, Wołowiec, 2020 (non traduit en français).

9. Å. Seierstad, En av oss: en fortelling om Norge [Un parmi nous : une histoire norvégienne], Oslo, Kagge, 2013 (non traduit en français).

10. B. Rawlence, City of Thorns. Nine Lives in the World's Largest Refugee Camp, Londres/New York, Picador, 2016 (non traduit en français).

11. B. Rawlence, ouvr. cité, (en polonais : Miasto cierni. Największy obóz dla uchodźców, traduit de l'anglais par S. Kowalski, Czarne, Wołowiec, 2017, p. 369).

12. Supplément de Gazeta Wyborcza, un des principaux quotidiens polonais, consacré au reportage.

13. M. Szczygieł, Nie ma [Il n'y a pas], Varsovie, Dowody na istnienie, 2018, p. 292-293 (non traduit en français).

14. P. P. Reszka, Płuczki. Poszukiwacze żydowskiego złota, Varsovie, Agora, 2019 (non traduit en français).

15. R. Barthes, «L'effet de réel », Communications, n 11, 1968, p. 84-89.

16. J'emprunte cette notion à Deleuze et Guattari tout en la dégageant de l'utilisation qu'ils en font. Voir G. Deleuze et F. Guattari, Kafka. Pour une littérature mineure, Paris, Les Éditions de Minuit, coll. « Critique », 1975.

17. A. Słonimski, «Listy z Ameryki. Chicago » [«Lettres d'Amérique. Chicago »], Wiadomości Literackie, $1936, \mathrm{n}^{\circ} 2$.

18. Å. Seierstad, L'Ange de Grozny, traduit du norvégien par L.-M. Besançon, Paris, Jean-Claude Lattès, 2008 [De krekede, 2007].

19. M. Caparrós, La Faim, traduit de l'espagnol par A. Carrasco, Paris, Buchet-Chastel, 2015 [El hambre, 2015].

20. M. Wasilewski, Jutro przypłynie królowa [La reine arrivera demain], Czarne, Wołowiec, 2020 (non traduit en français).

21. D. Varella, Carandiru, São Paolo, Brésil, traduit du portugais par S. Dosse, Éditions l'Aube, coll. « Document », 2005 [Estação Carandiro, 1999].

22. A. Russell Hochschild, Strangers in their own land: Anger and Mourning on the American Right, New York, The New Press, 2016 (non traduit en français). 
23. S. Drakulić, They would never hurt a fly, traduit du croate, New York, Little Brown, 2003 (non traduit en français).

24. H. Arendt, Eichmann à Jérusalem: rapport sur la banalité du mal, traduit de l'anglais par A. Guérin, Paris, Gallimard, coll. «Folio Histoire », 1991 [Eichmann in Jerusalem: A Report on the Banality of Evil, 1963]

25. E. D. Morel, King Leopold's Rule in Africa, Londres, William Heinemann, 1904 (non traduit en français).

26. Wiadomości Literackie [Les Nouvelles littéraires], 1933.

27. J. T. Gross, Les Voisins. 10 juillet 1941. Un massacre de Juifs en Pologne, traduit de l'américain par P.-E. Dauzat, Paris, Fayard, 2002 [Neighbors: The Destruction of the Jewish Community in Jedwabne, Poland, 2001].

28. W. Łukaszewski, MĄdrość i różne niemądrości [La Sagesse et quelques bêtises], Sopot, Smak Słowa, 2018, p. 242 (non traduit en français).

29. S. Sontag, Devant la douleur des autres, traduit de l'américain par F. Durand Bogaert, Christian Bourgois, 2003 [Regarding the Pain of Others, 2003].

30. A. Wójcińska, Perspektywa mrówki. Rozmowy z reporterami świata [Du point de vue de la fourmis. Entretiens avec des reporters du monde entier], Czarne, Wołowiec, 2015, p. 138 (non traduit en français).

31. G. Walraff, Tête de turc, traduit de l'allemand par A. Brossat et K. Schuffels, Paris, La Découverte, 1986 [Ganz unten, Kiepenheuer \& Witsch, Cologne, 1985].

32. «Unter null. Wie leben Obdachlose in Deutschland? » [ En dessous de zéro. Comment vivent les sans-abris en Allemagne »], Die Zeit, $\mathrm{n}^{\circ} 5$, mars 2009. On trouvera un choix de reportages de G. Wallraf en français dans le recueil Les perdants du meilleur des mondes, Paris, La Découverte, 2010 [Günter Wallraff Undercover. Reportagen aus der schönen neuen Welt, Berlin, Absolut Medien, 2010].

33. Ibid., p. 97.

34. L. Marx, «Pastorializm w Ameryce " [Le pastoralisme en Amérique], A. Preis-Smith (dir.), Kultura, tekst, ideologia. Dyskursy wspótczesnej amerykanistyki [Culture, texte, idéologie. Les discours des américanistes contemporains], Cracovie, Agata Preis-Smith, 2004, p. 96 (non traduit en français).

35. W. Jagielski, Nocni wĘdrowcy [Les vagabonds de la nuit], varsovie, Biblioteka Akustyczna, 2009, p. 323 (non traduit en français).

\section{RÉSUMÉS}

Cet article porte sur la littérature non fictionnelle. Il propose une théorie du reportage formée à partir de trois postulats. Le premier suppose que la littérature de ce genre est invérifiable et que le lecteur n'est pas en mesure de décider ce qui, dans le récit d'un reporter, représente le réel et ce qui est inventé par l'auteur. On pose également la question de la manière dont l'expérience réelle est transformée en récit littéraire. Le deuxième postulat spécifie que l'écriture de reportages implique la nécessité de quitter l'espace dont on est familier, c'est-à-dire une déterritorialisation. En ce sens, le reportage contemporain s'enracine dans la tradition de la littérature de voyage. Le troisième postulat interprétatif est le semi-perspectivisme du reportage, c'est-à-dire une prise de distance de l'auteur par rapport à son propre point de vue, une ouverture aux arguments des autres et une capacité à comprendre différentes perspectives. Les 
trois postulats sont proposés à partir d'une analyse de reportages contemporains écrits par des reporters de différentes aires culturelles.

The article deals with non-fiction literature. It is proposed the concept of interpreting this literary genre according to three keys. The first one assumes that this literature is unverifiable in terms of decisions, which in the reporter's account is a reflection of the reality. There is also about how real experience becomes fiction. The second issue says that reportage is connected with a kind of expansion, with the necessity of deterritorialization. It also refers to the tradition of apodemic literature. The third interpretative element is semi-perspectivism, meaning a specific writing attitude that requires for the author a distance from his own point of view. It also requires accepting the perspective of being open to the arguments of others. The three postulates are proposed on the basis of an analysis of contemporary reportage written by reporters from different cultural areas.

\section{INDEX}

Mots-clés : littérature contemporaine, reportage, journalisme littéraire

Keywords : contemporary literature, reportage, literary journalism

\section{AUTEURS}

\section{URSZULA GLENSK}

Université de Wrocław

Urszula Glensk est chercheuse en littérature, critique littéraire et professeur à l'université de Wrocław. Elle enseigne à l'Institut du Journalisme et de la Communication Sociale. Depuis 2019, elle fait partie du jury du prix littéraire d'Europe centrale Angelus. Elle a publié près de 200 articles scientifiques et dans la presse ainsi que cinq livres. Entre autres, la monographie Historia słabych. Reportaż $i$ życie w Dwudziestoleciu 1918-1939 [L'Histoire des faibles. Le reportage et la vie dans l'entre-deux-guerres (Universitas, 2014)] qui a obtenu le prix du livre d'histoire de l'hebdomadaire Polityka. Son dossier d'habilitation a été récompensé par le prix du Président du Conseil des Ministres. Son ouvrage le plus récent, Hirszfeldowie - zrozumieć krew [Les Hirschfeld: comprendre le sang (Universitas, 2019] a été nominé pour le prix Nike, et a obtenu le prix Kazimierz Moczarski (décernés par les Clubs historiques de la Jeunesse) ainsi que le prix Leopoldina. 